\title{
Gain Scheduling Control of a Bidirectional DC-DC Converter with Large Dead-Time
}

\author{
K. Engelen , S. De Breucker ${ }^{*}$, P. Tant and J. Driesen ${ }^{\circ}$ \\ Department of Electrical Engineering, ESAT-ELECTA, KU Leuven, Kasteelpark Arenberg 10, 3001 Heverlee, Belgium \\ (e-mail: kristof.engelen1@esat.kuleuven.be, peter.tant@esat.kuleuven.be, johan.driesen@esat.kuleuven.be). \\ Vito, Boeretang 200, $2400 \mathrm{Mol}$, Belgium (phone: +32 14 335827; fax: +32 14 321185; \\ e-mail: sven.debreucker@vito.be).
}

\begin{abstract}
This paper investigates the impact of the dead-time of the semiconductor switches on the operation of a dc-dc converter used in a bidirectional storage system for a hybrid electric vehicle. The dead-time of the switches of the dc-dc converter under investigation is exaggerated to clearly illustrate the effects of the non-linear dependency of the current on the duty cycle. The resulting current response makes it impossible for a simple PI-controller to regulate the current in a satisfactory way. In this paper the mechanism behind the highly non-linear behaviour is investigated. The results of the analysis are used to develop a control scheme that employs gain scheduling and duty cycle presetting to cope with the non-linear response of the converter current to changes in the duty cycle. Experimental results are presented based on the implementation of the proposed control algorithm on a two-phase interleaved bidirectional boost converter to prove the viability of the concept.
\end{abstract}

\section{Introduction}

In recent years a lot of research effort is focused on the development of high power density (HPD) bidirectional boost dc-dc power converters. These converters are well suited for storage applications in hybrid electric vehicles (HEVs) and smart grids. One of the main concerns in HPD converter design is the reduction of the size of the magnetic components. For most conventional converter topologies operating at moderate switching frequencies $(<100 \mathrm{kHz})$, this reduction leads to an increased peak-topeak value of the current and demands a faster control system. As a result, the converter will be more sensitive to dead-time related effects.

The mitigation of dead-time has been studied extensively for grid connected inverters and motor drives [1-8]. A feedback type dead-time compensation is applied in [1], by adjusting for the delay between the applied and actual duty cycle. The feedback is based on the measured output voltage timing. In [2] the output voltage distortion caused by the dead-time in vector-controlled motor drives is compensated. The compensation mechanism uses the sixth harmonic of the integrator output of the synchronous reference frame current controller. In [4] a feed-forward voltage is added to the output of the current controller to achieve dead-time compensation in a PMSM motor drive. A similar situation can be found in [5] and [8] where dead-time compensation is applied to induction motor drives to reduce the THD of the inverter voltage. In [3], a resonant current controller compensates the harmonic distortion of a grid connected inverter caused by the dead-time in discontinuous conduction mode (DCM). No current direction detection circuit is required. The dead-time is suppressed in DCM and eliminated in continuous conduction mode (CCM). [7] eliminates dead-time based on the instantaneous current direction, but 
requires a detection circuit. An overview of dead-time compensation, dead-time elimination and deadtime minimization methods for drive inverters can be found in [6].

[9] describes a compensation method for a dc-dc converter. No additional components or modifications of the gate driver are required. It does however require a constant alteration of the dead-time of both switches. The dead-time calculation algorithm requires several tens of switching cycles to find the optimum duty cycle and dead-time.

Most methods are designed for grid connected inverters and motor drives with sinusoidal currents and, due to the relatively large inductance of the motor or filter, a limited current ripple. Thus, in these applications DCM is limited to a small region of the total current range.

In this paper the goal is to realize the control scheme of a bidirectional boost converter without making any alterations to the hardware of the half-bridges or the gate drivers and without adding additional hardware for e.g. current direction detection. This control scheme thus allows the usage of common half-bridges, even when the dead-time is large compared to the switching period. In contrast to previous solutions, the operation of the converter in DCM over a relatively large part of the current range no longer poses a problem due to a thorough understanding of the effects of the dead-time. This allows the usage of a relatively small inductor.

In the investigated dc-dc converter, DCM is present in a large region of the current range. The current reference is no longer sinusoidal, but includes step changes. This requires a fast and stable controller able to deal with brisk transitions between DCM and CCM and a prolonged duration in DCM. Therefore a gain-scheduling current controller was designed for this small inductance dc-dc converter.

A series HEV was built for didactical and research purposes [20],[21]. The vehicle is equipped with a bidirectional dc-dc converter. The dead-time $\left(T_{d}\right)$ of the switches is purposely set at a value larger than strictly necessary $(2 \mu \mathrm{s})$, which is relatively large compared to the switching period $\left(T_{s w}\right)$ of $12.5 \mu \mathrm{s}$. The dead-time causes DCM. As the dead-time is relatively large, the discontinuity is present for a large range of duty cycle values. In CCM minor changes of the duty cycle are required to cover the entire current range. Section 4 provides an in-depth discussion of the non-linear behaviour of the converter current.

In DCM a PI-controller can be used for the regulation of the converter current. However, CCM requires a second set of PI-parameters. On the boundary between DCM and CCM, the PI-parameters must be changed by an additional control algorithm. The proposed control algorithm presets the duty cycle to a fixed value around the equilibrium value such that the current can change between DCM and CCM and between positive and negative continuous currents without the risk of instability or an excessively slow response.

\section{System description}

The dc-dc converter under investigation comprises two parallel bidirectional boost converters, each carrying a current of $30 \mathrm{~A}$. This topology is also applied in popular hybrids such as the Toyota Prius [19]. Multiphase interleaved converters of this type are the subject of recent academic research [15]-[17].

Each (sub-)converter consists of two controllable switches with anti-parallel diodes, connected in a half bridge configuration. The switching dynamics of the devices are not important. IGBTs are used in this converter, although other semiconductor devices such as MOSFETs could have been used as well. The low voltage input of each boost converter is connected to a $64 \mu \mathrm{H}$ inductor, while the high voltage output is connected to a $4 \mathrm{mF}$ capacitor which, in turn, is connected to the dc-bus of the motor inverter. 
To limit the current ripple, while keeping the size of the inductors relatively small, the switching frequency, $f_{s w}$, is set at $80 \mathrm{kHz}$. The volume-index $\left(\left.L \bullet\right|_{\text {peak }}{ }^{2}\right)$ of the inductor can be further reduced [18], but this causes high switch-off losses and requires a lossless capacitor snubber. A model of one boost converter is shown in Fig. 1a. A positive current is defined as a current flowing from the low voltage source into the midpoint of the IGBT half bridge.
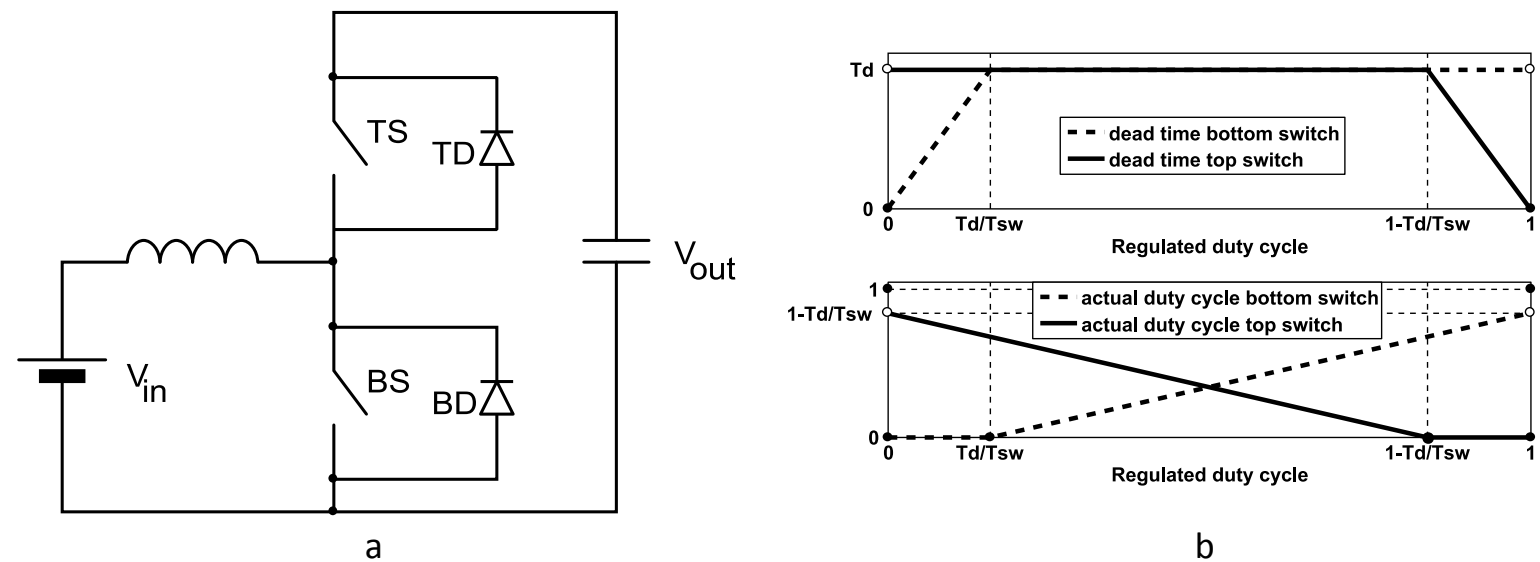

a

b

Fig. 1. Converter under investigation

a Converter schematic with current sequence; $\mathbf{b}$ Dead-time and $D_{\text {eff }}$ of the bottom and top switch, discontinuous implementation.

The switches are operated using complementary switching, i.e. the bottom switch state is complementary to the top switch state. A small amount of dead-time $\left(T_{d}\right)$ is needed to prevent simultaneous conduction of both switches (shoot-through).

The sampling frequency of the control scheme of the HEV is fixed at $10 \mathrm{kHz}$. As a consequence, the duty cycle of the switches will only be updated after 8 consecutive switching periods.

\section{Implementation of dead-time}

In order to make the following sections comprehensible three definitions of the duty cycle are given.

The regulated duty cycle $D_{\text {reg }}$ is the duty cycle of the bottom switch which is determined by the controller. The controlled part of the regulated duty cycle is indicated with $\delta$, the feed forward duty cycle is indicated with $D_{\mathrm{FF}}$, resulting in the formula $D_{\text {reg }}=D_{\mathrm{FF}}+\delta$. $D_{\text {reg }}$ ranges between 0 and 1.

$>$ The actual duty cycle $D_{\text {act }}$ of the bottom and top switch is defined as that part of the switching period during which the gate signals of respectively the bottom and top switch are high. The difference between $D_{\text {reg }}$ and $D_{\text {act }}$ is caused by the dead-time.

$>$ The effective duty cycle $D_{\text {eff }}$ of the bottom and top switch is defined as that part of the switching period during which the current is rising and falling, respectively. A non-zero voltage is then imposed across the inductor. $D_{\text {eff }}$ takes the conduction of the anti-parallel diodes into account.

The mentioned duty cycles refer to the duty cycle of the bottom switch unless stated otherwise. The figures in this section are based on an ideal converter, with a dead-time $\left(T_{d}\right)$ of $2 \mu \mathrm{s}$, a switching period $\left(T_{s w}\right)$ of $12.5 \mu s$ and no losses. The purpose of this section is to discuss the effect of dead-time on the duty cycle of an ideal converter. 
Dead-time can be implemented in several ways. In this paper a discontinuous implementation is considered. In Fig. 1b, the dead-time and the actual duty cycle of both the top and the bottom switch are shown as function of $D_{\text {reg. }}$. For values of $D_{\text {reg }}$ above $T_{d} / T_{s w}, 0.16$ in this case, the dead-time of the bottom switch is equal to $2 \mu \mathrm{s}$ until $D_{\text {reg }}$ reaches 1 . At this point the dead-time becomes $0 \mu \mathrm{s}$. The deadtime of the top switch is equal to $2 \mu \mathrm{s}$ when $D_{\text {reg }}$ is between $T_{d} / T_{s w}$ and $1-T_{d} / T_{s w}$. For values of $D_{\text {reg }}$ above $1-T_{d} / T_{s w}$, i.e. 0.84 , the dead-time of the top switch will decrease linearly from $2 \mu \mathrm{s}$ at a $\mathrm{D}_{\text {reg }}$ of $1-T_{d} / T_{s w}$ to $0 \mu$ s at a $D_{\text {reg }}$ of 1 .

\section{Effects of dead-time on duty cycle}

The effect of the dead-time on $D_{\text {act }}$ is illustrated in table I. This table shows the $D_{\text {reg, }}$ gate signals and $D_{\text {eff }}$ in the upper part of rows 3,5 and 7 for different values of $D_{\text {reg }}$ and current. The first gate signal is the gate signal of the bottom switch and the second, if present, that of the top switch. The lower part shows which of the elements are conducting the current; this can be the Bottom Switch (BS), Top Diode (TD), Top Switch (TS) or the Bottom Diode (BD). In all cases the input voltage is $96 \mathrm{~V}$, the output voltage is $400 \mathrm{~V}$ and the inductor has a constant inductance $(64 \mu \mathrm{H})$. In practice, the input and output voltages will deviate from these values, but in this example the values are fixed to allow a better comparison of the different situations. The resulting current waveform for each initial current and $D_{\text {reg }}$ is also illustrated in table I.

When no dead-time is implemented in the converter, the inductor current is continuous at all times. When the current evolves from a negative value to a positive value, the bottom switch takes over the current from its anti-parallel diode as it passes through zero. A similar statement can be made about the top switch. Therefore, for currents between $-I_{p, \text { ripple }}$ and $I_{p, \text { ripple }}$ (the peak value of the current ripple), also referred to as small currents, this converter operates under zero-voltage switching conditions [17]. In an ideal converter (i.e. no losses / voltage drops) with no dead-time, the steady-state current is independent of the duty cycle.

As stated, the converter described in this paper uses IGBT switches with an exaggerated dead-time to clearly illustrate the effect of a large $T_{d} / T_{s w}$ ratio. When the switching period is large compared to the dead-time, the aforementioned (ideal) behaviour can still be accurately approximated. Conversely, the behaviour of a converter operating with a large $T_{d} / T_{s w}$ ratio is affected profoundly by the dead-time. For a large range of duty cycle values there is a fixed relationship between duty cycle and steady-state current. Equation 9 of section $4 c$ gives the average current as function the regulated duty cycle $D_{\text {reg, }}$ which is illustrated in Fig. 3a. The dependency of the current on the duty cycle is caused by discontinuities in the current waveform. In case the average of the current is small, the current can remain zero during a part of the switching period.
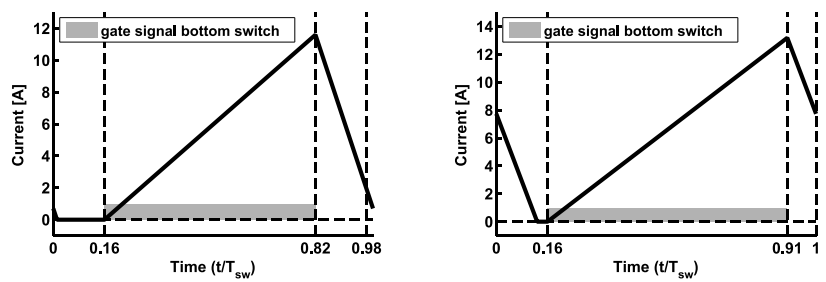

Fig. 2. Discontinuous currents due to dead-time for stable currents with $D_{\text {reg }}$ equal to 0.82 (left) and 0.91 (right). 
This is illustrated in Fig. 2 for small positive currents. In this figure, the inductor current is shown for two different values of the duty cycle. Both currents are stable for the chosen duty cycle, that is, if all parameters remain unchanged, the current does not change from one switching period to the next. For both duty cycles, the current starts to increase from zero when the bottom switch is turned on. When the bottom switch is turned off, the current starts to free-wheel through the top diode. The current returns to zero during the dead-time interval preceding the on-time of the bottom switch. In the right graph of Fig. 2 the duty cycle is significantly larger than in the left graph, however, the average current has only increased marginally. The peak value of the current is larger on the right, and the top diode conducts for a longer period of time. Thus, in this situation, the current will barely react on changes in the duty cycle.

\section{a. Effects of dead-time on continuous currents}

Above a certain $D_{\text {reg }}$ the current becomes continuous. In case the average current is positive, only the bottom switch and top diode will conduct as shown in table I (pos. currents, $D_{\text {reg }}=0.92$ ). For a negative continuous current, only the top switch and the bottom diode will conduct, as shown in table I (neg. currents, $D_{\text {reg }}=0.6$ ). As soon as the current becomes continuous, it reacts strongly to small changes in the duty cycle (Fig. 3). When the duty cycle changes, the average inductor voltage will become non-zero. Because of the small inductance value, the average inductor current will change rapidly. In a non-ideal converter there are various sources of current dependent voltage drops, such as the forward voltage drop across the IGBTs and the equivalent series resistance of the inductor, which tend to counteract the change in average inductor voltage. The average inductor voltage will eventually return to zero. These voltage drops are preferably very small, which explains why the resulting change in current will be very large.

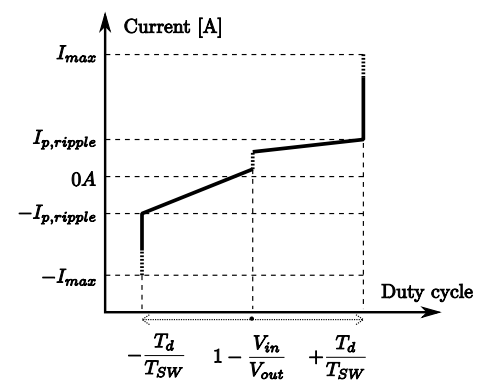

a

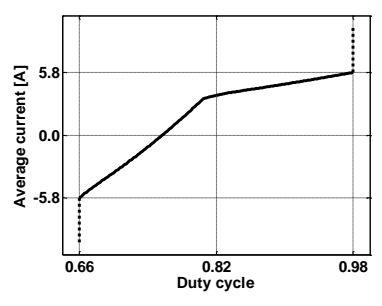

C

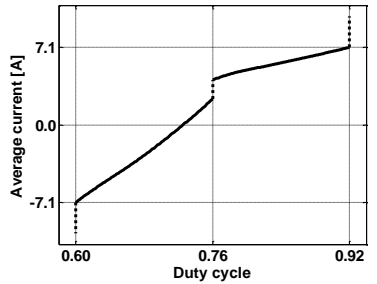

b

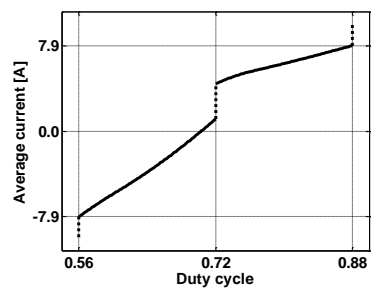

d

Fig. 3. Dependency of current on duty cycle.

a General ; b $V_{\text {IN }}=96 \mathrm{~V}, \mathrm{~V}_{\text {OUT }}=400 \mathrm{~V} ; \mathbf{c} \mathrm{V}_{\text {IN }}=72 \mathrm{~V}, \mathrm{~V}_{\text {OUT }}=400 \mathrm{~V} ; \mathbf{d} \mathrm{V}_{\text {IN }}=112 \mathrm{~V}, \mathrm{~V}_{\text {OUT }}=400 \mathrm{~V}$

According to the formula $D=1-V_{\text {in }} / V_{\text {out }}$, which is readily obtained from the volt-second balance of the inductor [22], a stable current is achieved in this example when the duty cycle is 0.76 . If we apply this $D_{\text {reg }}$ to e.g. a positive initial current of $15 \mathrm{~A}$ (table I, pos. current, $D_{\text {reg }}=0.76$ ), the current flows through the top diode during the dead-time of the bottom switch and not through the bottom switch as would 
be the case if no dead-time were implemented. This causes $D_{\text {eff }}$ to be smaller than $D_{\text {reg }}$ by a value equal to $T_{d} / T_{s w}(0.16)$ and results in a decreasing current. In order to obtain a stable, positive current, $\mathrm{D}_{\text {eff }}$ must correspond to the equilibrium value of $1-V_{\text {in }} / V_{\text {out }}(0.76)$. This is achieved when $D_{\text {reg }}$ is equal to $1-V_{\text {in }} / V_{\text {out }}+T_{d} / T_{\text {sw }}$ (table I, pos. current, $D_{\text {reg }}=0.92$ ). All positive continuous currents of the actual converter will have a $D_{\text {reg }}$ that only deviates in the order of magnitude of 0.01 from this value to compensate amongst others for the resistance of the wires.

For a negative initial current of e.g. $-15 \mathrm{~A}$, a $\mathrm{D}_{\text {reg }}$ of 0.76 will result in a $\mathrm{D}_{\text {eff }}$ of 0.8 and an increasing current. $D_{\text {eff }}$ is larger than $D_{\text {reg }}$ due to the conduction of the current through the bottom diode during the dead-time of the top switch (table I, neg. current, $D_{\text {reg }}=0.76$ ). In order to obtain a stable negative current, the portion of the switching period occupied by the dead-time of the top switch, $T_{d} / T_{s w}$, must be subtracted from $D_{\text {reg. }}$. The resulting $D_{\text {reg }}$ of $1-V_{\text {in }} / V_{\text {out }}-T_{d} / T_{\text {sw }}(0.6)$ will result in a $D_{\text {eff }}$ of $1-V_{\text {in }} / V_{\text {out }}$ and a stable negative current. For the same reasons as positive continuous currents, all negative continuous currents of the actual converter will have a $D_{\text {reg }}$ that only deviates in the order of magnitude of 0.01 from $1-V_{\text {in }} / V_{\text {out }}-T_{d} / T_{\text {sw }}$.

In summary, it can be stated that, in order to obtain a stable positive or negative continuous current, $D_{\text {eff }}$ will have to be close to the theoretical equilibrium value of $1-V_{\text {in }} / V_{\text {out }}$. This means that $\mathrm{D}_{\text {reg }}$ will have to be close to $1-V_{\text {in }} / V_{\text {out }} \pm T_{d} / T_{\text {sw }}$.

In practice, the equilibrium value will be implemented as $D_{\mathrm{FF}}$, the output of a feed forward controller. If the input and output voltage of the converter change, the equilibrium value $D_{\mathrm{FF}}$ will change as well, but the value that has to be added to, or subtracted from, $D_{\mathrm{FF}}$ to achieve a stable negative or positive current remains the same.

\section{a. Discontinuous currents}

All values of $\mathrm{D}_{\text {reg }}$ that lie between $1-V_{\text {in }} / V_{\text {out }}-T_{d} / T_{s w}$ and $1-V_{\text {in }} / V_{\text {out }}+T_{d} / T_{s w}$, will result in a discontinuous steady state current. Whereas the $D_{\text {reg }}$ of continuous currents deviates an order of magnitude of 0.01 , the duty cycle range for the near-zero currents reaches $2 \cdot T_{d} / T_{s w}$, i.e. a staggering 0.32 in this case. This is due to the fact that the combined duration of the dead-time of both switches occupies $32 \%$ of the switching period. The controller for discontinuous currents needs a substantial increase in range to cover all possible $D_{\text {reg }}$ compared to the controller for continuous currents.

A stable, small, positive current, with an initial value of $4.8 \mathrm{~A}$, is shown in table I (near-zero currents, $\left.D_{\text {reg }}=0.86\right)$. The current increases between the points in time corresponding to $0.16 \cdot T_{s w}$ and $0.86 \cdot T_{s w}$, resulting in a $D_{\text {eff }}$ of 0.7 for the bottom switch. This seems to contradict the expected $D_{\text {eff }}$ of 0.76 for a stable current. However, due to the discontinuity in the current waveform, $D_{\text {eff }}$ of the top switch (0.221) is not complementary with $D_{\text {eff }}$ of the bottom switch. For steady state operation it is required that the average voltage across the inductor, given by $V_{\text {ind, avg }}=V_{\text {in }} \cdot D_{\text {eff }}+\left(V_{\text {in }}-V_{\text {out }}\right) \cdot D_{\text {eff, },}$, equals zero (volt-second balance). The condition for steady state operation, $V_{\text {ind,avg }}=0$, can be written as $D_{\text {eff }} /\left(D_{\text {eff }}+D_{\text {eff, }, T}\right)=1-V_{\text {in }} / V_{\text {out }}$. In the example, the ratio of $D_{\text {eff }}$ and the sum of the effective duty cycles equals 0.76 , which explains why the current in this example is stable.

All stable discontinuous currents require the same ratio between $D_{\text {eff }}$ of the bottom switch and the sum of both $D_{\text {eff }} S$, which is equal to $1-V_{\text {in }} / V_{\text {out }}$. 


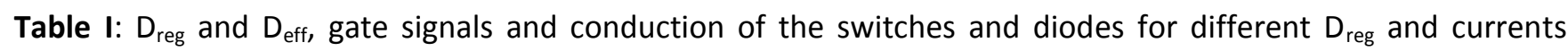
together with the corresponding current profile.

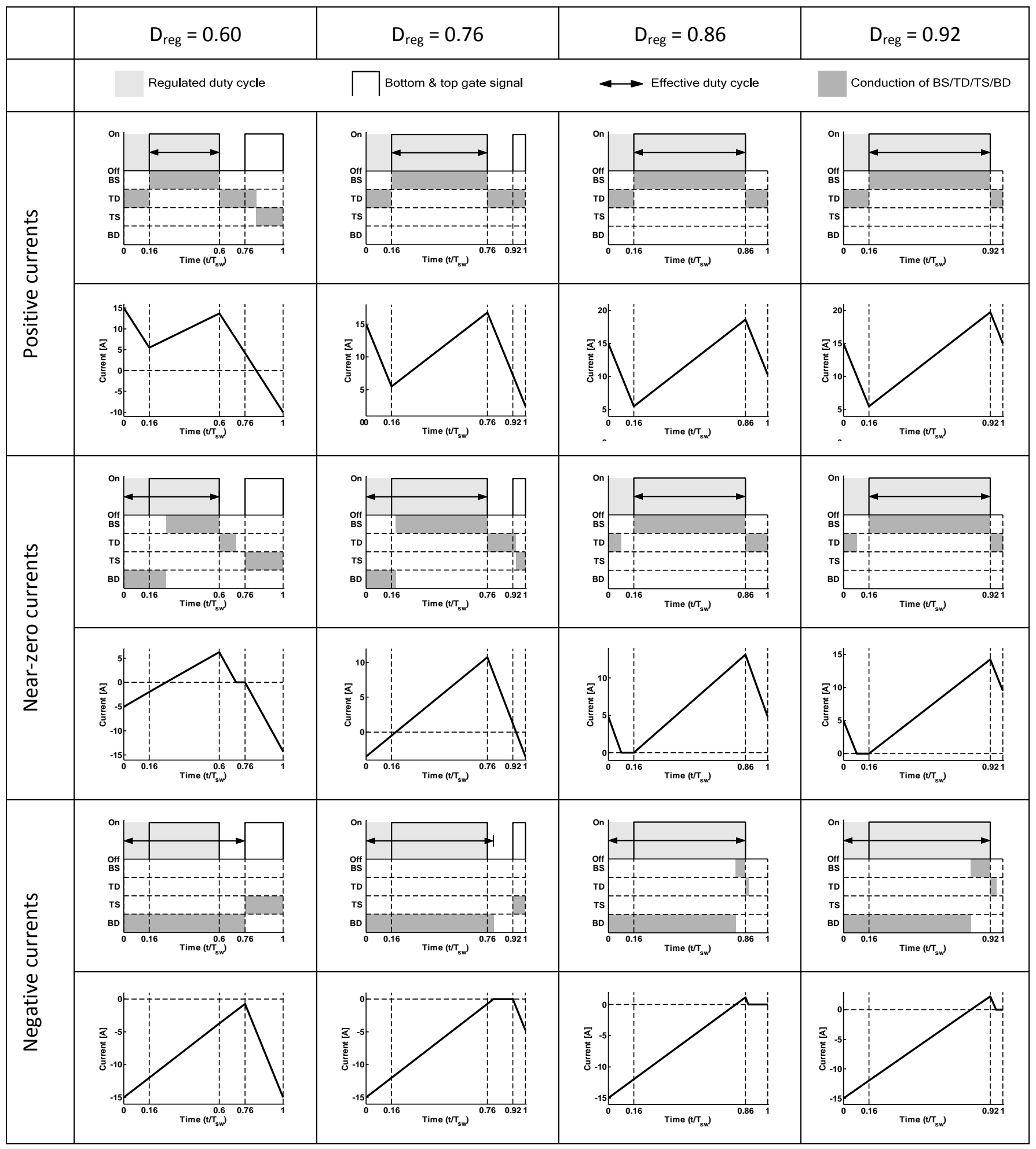


A stable, small current with negative initial value is illustrated in table I (near-zero currents, $D_{\text {reg }}=0.76$ ). Although $D_{\text {reg }}$ has dropped from 0.86 to 0.76 , the RMS-current has only changed from $6.5 \mathrm{~A}$ to $4.2 \mathrm{~A}$, clearly indicating that a large change of the duty cycle has little effect on discontinuous currents. Deviations from the correct duty cycle will not cause the current to reach excessive values as long as $D_{\text {reg }}$ stays between $1-V_{\text {in }} / V_{\text {out }}-T_{d} / T_{\text {sw }}$ and $1-V_{\text {in }} / V_{\text {out }}+T_{d} / T_{s w}$. It should be noted that $D_{\text {eff }}$ of a small current with negative initial value includes the dead-time of the bottom switch, which is not the case for $D_{\text {eff }}$ of a small current with positive initial value.

\section{b. Average current in discontinuous current mode.}

In order to calculate the average value of the current as function of $D_{\text {reg, }}$, five distinct discontinuous current waveforms are identified, each of which is determined by the polarity of the current and the interval where the discontinuity appears. The boundaries of the regulated duty cycle range for each type of discontinuous current as function of the voltage ratio between the battery and dc-bus are determined.

Unipolar positive current, discontinuity in dead-time interval of $B S$. $\left(B_{3} \leq D_{\text {reg }} \leq D_{n}+T_{d} / T_{s w}\right)$

Unipolar negative current, discontinuity in dead-time interval of TS. $\left(D_{n}-T_{d} / T_{s w} \leq D_{\text {reg }} \leq B_{4}\right)$

Bipolar current, discontinuity in dead-time interval of $B S$. $\left(B_{1} \leq D_{\text {reg }} \leq B_{3}\right)$

Bipolar current, discontinuity in dead-time interval of TS. $\left(B_{4} \leq D_{\text {reg }} \leq B_{2}\right)$

Bipolar current, discontinuity in dead-time interval of both TS and BS. $\left(B_{2} \leq D_{\text {reg }} \leq B_{1}\right)$

In these equations $D_{n}$ equals 1- $\left(V_{\text {in }} / V_{\text {out }}\right)$. Depending on the value of $D_{n}$, different types of discontinuous currents exist. The boundaries of the duty cycle range for each type of discontinuity are given in equations 1 to 4 .

$$
\begin{aligned}
& B_{1}\left(D_{n}\right)=\min \left\{1-T_{D} / T_{S W}, \max \left\{D_{n}, \frac{T_{D} / T_{S W}}{1-D_{n}}\right\}\right\} \\
& B_{2}\left(D_{n}\right)=\min \left\{T_{D} / T_{S W}, \max \left\{D_{n}, \frac{1-T_{D} / T_{S W}}{D_{n}}\right\}\right\} \\
& B_{3}\left(D_{n}\right)=\min \left\{\left(1-T_{D} / T_{S W}\right) D_{n}+T_{D} / T_{S W}, 1-T_{D} / T_{S W}\right\} \\
& B_{4}\left(D_{n}\right)=\max \left\{\left(1-T_{D} / T_{S W}\right) D_{n}, T_{D} / T_{S W}\right\}
\end{aligned}
$$

The average current can be determined using equation 9. This equation uses the variables $x_{1}$ to $x_{4}$ as defined by equations (5) to (8) respectively. These equations are only valid for the specified range of $D_{\text {reg. }}$. Outside of this range, the variables $x_{1}$ to $x_{4}$ are equal to zero.

$$
\begin{aligned}
& x_{1}\left(D_{r e g}\right)=\frac{D_{r e g}-T_{D} / T_{S W}}{D_{n}}, \quad B_{2}\left(D_{n}\right) \leq D_{r e g} \leq D_{n}+T_{D} / T_{S W} \\
& x_{2}\left(D_{r e g}\right)=\frac{1-D_{r e g}-T_{D} / T_{S W}}{1-D_{n}}, \quad D_{n}-T_{D} / T_{S W} \leq D_{r e g}<B_{1}\left(D_{n}\right) \\
& x_{3}\left(D_{r e g}\right)=\frac{1-T_{D} / T_{S W}-x_{2}\left(D_{r e g}\right)}{D_{n}}, \quad B_{4}\left(D_{n}\right) \leq D_{\text {reg }}<B_{2}\left(D_{n}\right)
\end{aligned}
$$


$x_{4}\left(D_{r e g}\right)=\frac{1-T_{D} / T_{S W}-x_{1}\left(D_{r e g}\right)}{1-D_{n}}, \quad B_{1}\left(D_{n}\right) \leq D_{r e g} \leq B_{3}\left(D_{n}\right)$

$I\left(D_{\text {reg }}\right)=\sum_{k=1}^{4}(-1)^{k+1} x_{k}^{2}\left(D_{\text {reg }}\right) I_{\text {p,ripple }}$

A graphical representation of the average current as function of the regulated duty cycle is given in Fig. $3 b-d$ for three different battery voltages.

\section{Distinction between small and large currents}

A distinction can be made between large and small currents. In this particular case, small currents are those currents that are discontinuous, resulting in a current that barely reacts on changes in the duty cycle. On the other hand, large currents are continuous currents and are extremely sensitive to changes in the duty cycle. The distinction between small and large currents is determined by the boundary between continuous and discontinuous currents. This is further explained for positive currents. The peak-to-peak value of the current through the inductor is determined by $I_{\text {P2P }}=V_{\text {in }} \cdot\left(V_{\text {out }}-V_{\text {in }}\right) /\left(L \cdot f_{s w} \cdot V_{\text {out }}\right)$. The measured current equals the average current over one switching period. At the boundary between DCM and CCM, the lower peak of the triangular current waveform is at zero and the upper peak is equal to $I_{\text {P2P. }}$. This corresponds to a measured value of $I_{P 2 P} / 2$. Thus, the boundary between small and large currents is given by $I_{\text {boundary }}=V_{\text {in }} \cdot\left(V_{\text {out }}-V_{\text {in }}\right) /\left(2 \cdot L \cdot f_{\text {sw }} \cdot V_{\text {out }}\right)$.

\section{Gain scheduling controller with DCPA design}

Due to the extremely non-linear response of the current to changes in the duty cycle, the current cannot be controlled with sufficient accuracy by a simple PI-controller at $10 \mathrm{kHz}$. On the one hand it is possible to design a fast PI-controller that copes with the currents in the vicinity of zero, but the response of this controller for large currents is unstable. On the other hand it is possible to design a mild PI-controller that copes with fast varying, i.e. larger currents, but this controller is unacceptably slow for small currents. In order to cope with this problem a gain scheduling controller [11]-[13] is developed.

The first step of the proposed solution is to design a current controller that can regulate the currents with an absolute (measured) value below the amplitude $I_{\text {,ripple }}$ of the current ripple. For this a PIcontroller with anti-windup (AW, Fig. 4) is used. This controller needs to be very fast to compensate for the slow reacting system, i.e. the controller changes the duty cycle within a range of $2 \cdot T_{d} / T_{s w}$ in the

region between $-I_{p, \text { ripple }}$ and $I_{p, \text { ripple. }}$. Currents above $I_{p, \text { ripple }}$ cannot be regulated by this controller, resulting in overcurrents which trip the safety settings. In [10],[14] a state-space averaged model of a bidirectional boost converter is made, and pole placement is used to determine the control gains. However, due to the highly non-linear behaviour of the investigated converter, an accurate dynamic model is not readily obtained. Therefore, manual tuning of the PI controller is preferred. The practical implementation of the PI controller is elaborated upon in Section 8.

The next step is to design a second current controller of the same configuration as the first one, which

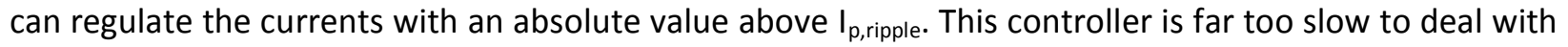
the currents below $I_{p}$,ripple. The anti-windup of this controller limits the integrator part of $\delta$ to a value slightly above $T_{d} / T_{s w}$ or below $-T_{d} / T_{s w}$. This does not interfere with the operation of the PI controller for small currents, as the $\delta$ for small currents will have an absolute value below $T_{d} / T_{s w}$. 
The final step is to make an algorithm, the Duty Cycle Preset Algorithm (DCPA), that determines which of the controllers should be used and brings $\delta$ in the vicinity of the correct $\delta$. As explained in section 4 , a change of the set point to a positive continuous current requires $D_{\mathrm{FF}}$ to be augmented by $T_{d} / T_{s w}$ to obtain a $D_{\text {eff }}$ of $1-V_{\text {in }} / V_{\text {out }}$, whereas a change of the set point to a continuous negative current requires $\mathrm{D}_{\mathrm{FF}}$ to be reduced by $T_{d} / T_{s w}$ to obtain a $\mathrm{D}_{\text {eff }}$ of $1-V_{\text {in }} / V_{\text {out }}$. The algorithm first determines the current value of $\delta$. If $\delta$ is below $-T_{d} / T_{s w}$, the current is considered large and negative, between $-T_{d} / T_{s w}$ and 0 the current is considered small and negative, between 0 and $T_{d} / T_{s w}$ the current is considered small and positive and above $T_{d} / T_{s w}$ the current is considered large and positive. The decision algorithm is illustrated in Fig. 4.

The first part of the decision algorithm determines the set-point $\delta_{S P}$ of $\delta$ for small currents. Based on the current value of $\delta$, the algorithm will first determine whether the present current is a large positive or a large negative current. In a next step, the algorithm will reset the integrator of the l-action to the value $\delta_{S P}$ based on the sign of the set-point $I_{\text {Ref }}$ and taking into account the magnitude of the current error $\varepsilon$.

The second part of the algorithm determines the set-point of $\delta$ for large currents. The algorithm first uses the current value of $\delta$ to determine if the current is small or of an opposite sign of the current reference $I_{\text {Ref. }}$. If this is the case and $I_{\text {Ref }}$ indicates that the set-point of the current is changed into a large current, the algorithm will intervene by resetting the integrator of the l-action. However, it will only do so if the absolute value of the error $\varepsilon$ is larger than $2 \mathrm{~A}$. This avoids unnecessary interventions of the DCPA.

The third part of the algorithm determines which P- and I-values of the PI-controller are used, based on the set-point of the current. This is illustrated in Fig. 4 by the variable $C_{S P}$ which selects the appropriate $\mathrm{P}$ - and I-values. When the set-point of the current changes to a small or large value, the P- and I-values turn the controller into a fast or mild controller, respectively.

\section{Influence of inductance value and input voltage level on the DCPA}

The boundary between continuous and discontinuous mode depends on the value of the inductance and the input voltage, and is given by $I_{\text {boundary }}=V_{\text {in }} \cdot D_{\text {eff }} /\left(2 \cdot L \cdot f_{\text {sw }}\right)$ (section 5$)$. In practice the influence of the inductance is limited and the input voltage will have a larger impact. In Fig. 3b-d, the relationship between the regulated duty cycle and the average current, as determined in equation 9 , is illustrated for 3 different input voltages.

When the actual boundary is lower than the estimated boundary of $7 \mathrm{~A}$, this will result in current oscillations (Fig. 5). Luckily the oscillations occur at modest current levels and do not trip the safety settings. When required, the influence of the voltage levels could be mitigated by using the voltage

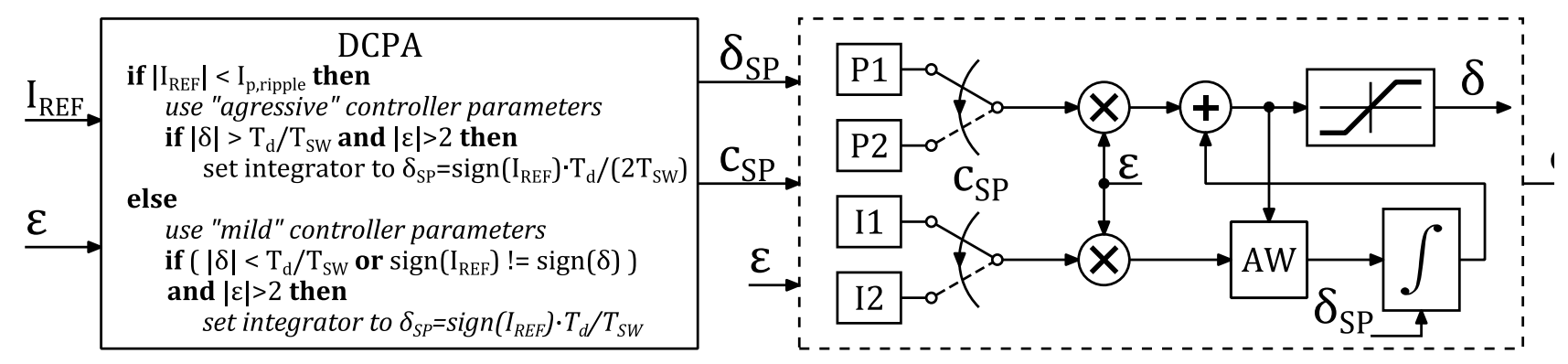

Fig. 4. Current controller with DCPA, gain scheduling and anti-windup. 
measurements to continuously update the boundary level. These oscillations can still occur at the exact current value of the boundary. However, this falls beyond the scope of the current study.

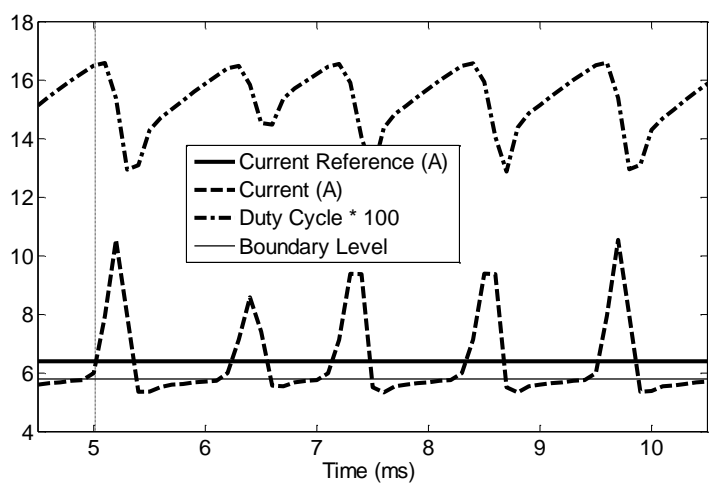

Fig. 5. Current oscillations.

\section{Experimental results}

In practice, a two-phase interleaved bidirectional boost converters is implemented to control the power flow between the battery and dc-bus of the motor inverter. The inductor of each phase is constructed with two PM62/49 ferrite cores and has an inductance value of approximately $64 \mu \mathrm{H}$. Both phases have a separate current controller of the same type and with the same parameters. The boundary between small and large currents is set at $7 \mathrm{~A}$. Both current controllers receive their set point from the dc-bus voltage controller, i.e. the voltage and current controllers form a cascade control system [21]. Both converters are equipped with 2 Fairchild HGTG30N60A4D IGBTs that can block up to $600 \mathrm{~V}$ and conduct up to $35 \mathrm{~A}$ at $80 \mathrm{kHz}$. The control system is implemented in Simulink and is subsequently executed on a computer running a real-time Linux operating system. Data acquisition and gate driver interfacing is implemented on an FPGA. Three tests are conducted to prove the correct functioning of the current controller with DCPA.

In order to obtain the PI-controller gains by manual tuning both input and output of the converter are connected to a controlled voltage source. This allows to determine the parameters of the current controller in absence of a voltage controller. After an initial estimation, first the P-value of the controller is fine-tuned based on the step-response of the current, subsequently followed by the fine-tuning of the I-value of the controller. The values of $P$ and $I$ are set at $1.5 e-3 A^{-1}$ and $8 A^{-1} S^{-1}$ respectively for the small current $\mathrm{PI}$-controller. These values are much smaller for the large current $\mathrm{PI}$-controller, with $\mathrm{P}$ - and $\mathrm{I}$ values of $4.5 \mathrm{e}-4 \mathrm{~A}^{-1}$ and $0.4 \mathrm{~A}^{-1} \mathrm{~s}^{-1}$ respectively. This controller is far too slow to deal with the currents below the $7 \mathrm{~A}$ boundary between discontinuous and continuous currents; $\mathrm{A}$ step change from 0 to $5 \mathrm{~A}$ takes the fast controller for small currents 5.5 ms to reach $4.5 \mathrm{~A}$, where the mild controller for large currents needs $60 \mathrm{~ms}$ to reach the same current value.

The first test is a step change of the current reference from $-5 \mathrm{~A}$ to $30 \mathrm{~A}$ and back to $-5 \mathrm{~A}$ (Fig. 6a). As soon as the step change of the reference value is applied, the DCPA resets the I-action of the PIcontroller (i.e. part of $\delta$ ) from approximately -0.16 to +0.16 , while the PI-controller adds an additional 0.02 to $\delta$. This step change of $\delta$ is clearly visible in the figure and causes the current to jump from $-5 \mathrm{~A}$ to $12 \mathrm{~A}$, after which the PI-controller adjusts $\delta$ to enable the current to rise to the $30 \mathrm{~A}$ reference value in $10 \mathrm{~ms}$. The $\delta$ originating from the PI-controller is added to $D_{\mathrm{FF}}$ to produce the total duty cycle. The step 
change back to $-5 \mathrm{~A}$ will occur in a similar fashion: the current jumps to $5 \mathrm{~A}$, after which it is adjusted to the -5 A reference value.

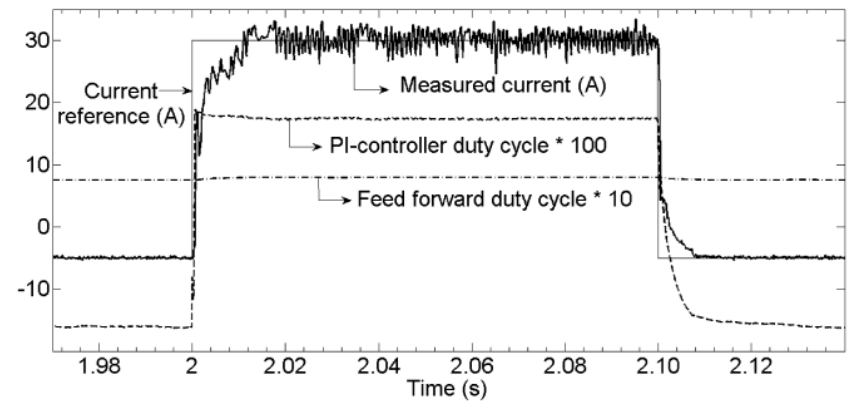

a

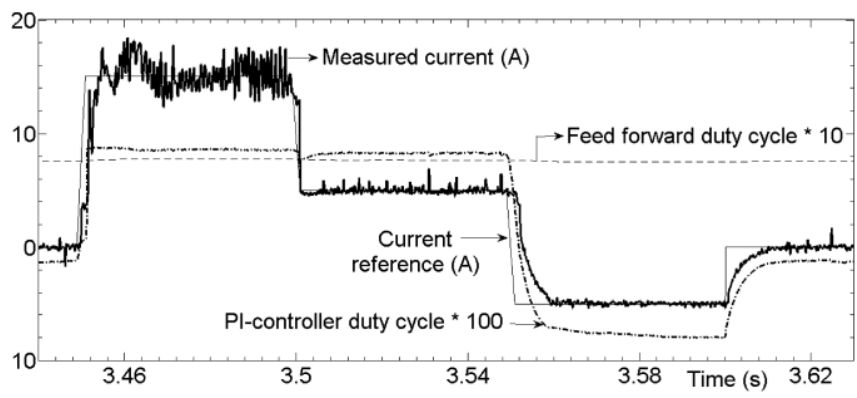

b

Fig. 6. Response on step change in current reference a from $-5 \mathrm{~A}$ to $30 \mathrm{~A}$ and back to $-5 \mathrm{~A}$; $\mathbf{b}$ from $0 \mathrm{~A}$ to $+15 \mathrm{~A}$ over $+5 \mathrm{~A}$ to $-5 \mathrm{~A}$.

A second test (Fig. 6b) examines smaller steps in the current reference; a first step from $0 \mathrm{~A}$ to $15 \mathrm{~A}$, a second step to $5 \mathrm{~A}$, a third step to $-5 \mathrm{~A}$ and back to $0 \mathrm{~A}$. In the first two steps the current reference passes the $7 \mathrm{~A}$ boundary between large and small currents and the DCPA will cause the current to jump to the vicinity of its set-point. In the last two steps there is no need for the DCPA to intervene and the PIcontroller will adjust the current until it reaches its set-point.

Fig. 7 shows the application of the dc-dc converter in a series hybrid vehicle, where the converter is back-to-back connected to the inverter of an induction motor. The dc-bus voltage control is realized by a cascade controller with inner current control loop and outer voltage control loop. The induction motor accelerates from standstill at full throttle. This translates in a power demand that rises from 0 to $4.5 \mathrm{~kW}$. The two-phase interleaved converter draws about $50 \mathrm{~A}$ ( $25 \mathrm{~A}$ per phase) out of the battery. Due to the fast response of the current controllers, the maximal deviation from the $400 \mathrm{~V}$ dc-bus set-point is $20 \mathrm{~V}$, which is more than satisfactory.

The tests prove that the DCPA allows the current to change rapidly without being hindered by the nonlinear response of the current on $D_{\text {reg. }}$. The tests also reveal that the two separate controllers for large and small currents are very effective. When the current reference changes from $5 \mathrm{~A}$ over $-5 \mathrm{~A}$ to $0 \mathrm{~A}$, the current will reach its set-point in $10 \mathrm{~ms}$, while the large currents reach their set-points in the same timespan. The large currents oscillate round their set-point due to the poor resolution of $D_{\text {reg, }}$ which is limited to 0.002 . This causes the current to switch between two distinct values, e.g. a $15 \mathrm{~A}$ current will 
switch between $14 \mathrm{~A}$ and $16 \mathrm{~A}$. The performance of the proposed current controller enables fast voltage regulation of the dc-bus when used in conjunction with a simple PI voltage controller.

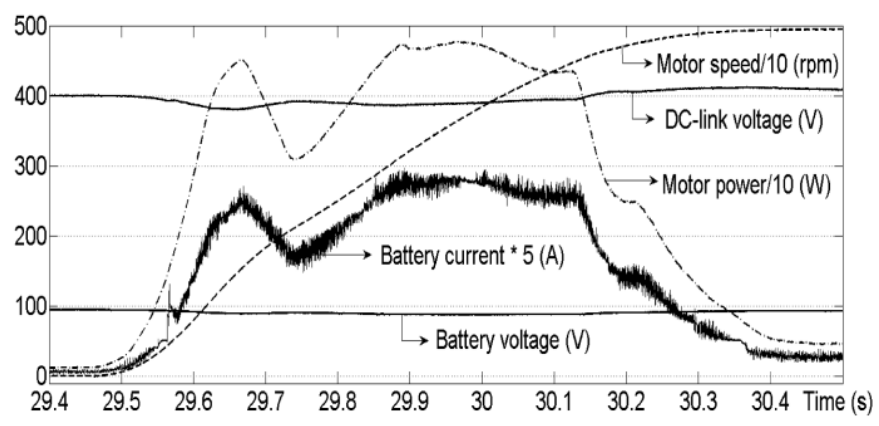

Fig. 7. Response of dc-bus voltage and battery current on full acceleration of an induction motor

\section{Conclusion}

This paper investigates the consequences of a high ratio of dead-time to switching period. In the case of a bidirectional boost converter with a relatively small dead-time, the current can be assumed to be continuous at all times and a simple PI-controller is sufficient to control the entire current range. When the dead-time is relatively large compared to the switching period, the current becomes discontinuous for all currents with a dc-value between $-I_{p}$,ripple and $I_{p}$,ripple. The duty cycle of these currents occupies $2 \cdot T_{d} / T_{s w}$, e.g. $32 \%$ in this case, of the duty cycle range. Currents below $-I_{p \text {,ripple }}$ and above $I_{p, \text { ripple }}$ are continuous and only require small changes of the duty cycle to span the entire continuous currents range. As both the boundary between continuous and discontinuous currents and the duty cycle deviation with respect to the equilibrium duty cycle $D_{\mathrm{FF}}$ at this boundary are known, a control algorithm (DCPA) is designed that presets the duty cycle depending on the reference value of the current. The DCPA is combined with a gain scheduling PI-controller, such that the PI-parameters can be adapted when the current changes between continuous and discontinuous mode. Tests prove that this method provides a stable and fast current controller. The current controller is based on the one hand on known characteristics of the dc-dc converter such as the dead-time, switching period and current ripple and on the other hand on well established controller architectures, such as the PI-controller. This allows the easy and quick adaptation of the current controller. The viability of the gain scheduling controller is proven by its implementation on a two-phase interleaved dc-dc converter which is back-to-back connected to the induction motor of a series hybrid vehicle.

\section{References}

[1] Masashi, O., Satoshi, O., and Masatsugu, T.: 'A Feedback-Type Dead-Time Compensation Method for High-Frequency PWM Inverter - Delay and Pulse Width Characteristics', Proc. $27^{\text {th }}$ Annu. IEEE Appl. Power Elec. Conf. and Exposition (APEC), Orlando, Florida, 5-9 Feb. 2012, pp. 100-105

[2] Hwang, S.-H., and Kim, J.-M.: 'Dead Time Compensation Method for Voltage-Fed PWM Inverter', IEEE Trans. Energy Conversion, March 2010, 25, (1), pp. 1-10

[3] Wang, Y., Gao, Q., and Cai, X.: 'Mixed PWM for Dead-Time Elimination and Compensation in a Grid-Tied Inverter', IEEE Trans. Ind. Electronics, Oct. 2011, 58, (10), pp. 4797-4803

[4] Kim, S.-Y., Lee, W., Rho, M.-S., and Park, S.-Y.: 'Effective Dead-Time Compensation Using a Simple Vectorial Disturbance Estimator in PMSM Drives', IEEE Trans. Ind. Electronics, May 2010, 57, (5), pp. $1609-1614$ 
[5] Oliveira, A. C., Jacobina, C. B., and Lima, A. M. N.: 'Improved Dead-Time Compensation for Sinusoidal PWM Inverters Operating at High Switching Frequencies': IEEE Trans. Ind. Electronics, Aug. 2007, 54, (4), pp. 2295-2304

[6] Lin, Y.-K., and Lai, Y.-S.: 'Dead-Time Elimination of PWM-Controlled Inverter/Converter Without Separate Power Sources for Current Polarity Detection Circuit', IEEE Trans. Ind. Electronics, June 2009, 56, (6), pp. 2121-2127

[7] Cho, K. M., Oh, W. S., Kim, Y. T., and Kim, H. J.: 'A New Switching Strategy for Pulse Width Modulation (PWM) Power Converters', IEEE Trans. Ind. Electronics, Feb. 2007, 54, (1), pp. 330-337

[8] Patel, P.J., Patel, V., and Tekwani, P.N.: 'Pulse-based dead-time compensation method for selfbalancing space vector pulse width-modulated scheme used in a three-level inverter-fed induction motor drive,' IET Power Electronics, July 2011, 4, (6), pp. 624-631

[9] Yousefzadeh, V., and Maksimovic, D.: 'Sensorless optimization of dead times in DC-DC converters with synchronous rectifiers', IEEE Trans. Power Electronics, July 2006, 21, (4), pp. 994- 1002

[10] Montagner, V.F., and Ferreira, A.A: 'Design and robustness analysis of a state feedback controller applied to a bidirectional boost converter', Proc. $10^{\text {th }}$ Brazilian Power Electronics Conference (COBEP '09), Bonito-Mato Grosso do Sul, Brazil, Sept. 27 - Oct. 1 2009, pp. 385-388

[11] Peng, H., and Chang, C.: 'A simple nonlinear gain scheduling method in digital PWM converter control', Proc. IEEE $6^{\text {th }}$ Int. Power Electr. and Motion Control Conf. (IPEMC '09), Wuhan, China, 17-20 May 2009, pp. 331-336

[12] Tseng, K.-H., and Chen, C.-L, 'Design and hardware implementation for a full-bridge phase-shift PWM DC/DC converter system with FPGA-based PI gain-scheduling control', Proc. $6^{\text {th }}$ IEEE Conference on Ind. Electr. and Applications (ICIEA), Beijing, China, 21-23 June 2011, pp. 1578-1582

[13] Su, J.-T., and Liu, C.-W.: 'Proposed digital control scheme for improved current share of multiphase DC/DC converters', Proc. IEEE $8^{\text {th }}$ Int. Conf. on Power Electronics and ECCE Asia (ICPE \& ECCE), Jeju, Korea (South) , May 30 - June 3 2011, pp. 1612-1617

[14] Xu, H. , Wen, X., Qiao, E., Guo, X., and Kong, L.: 'High Power Interleaved Boost Converter in Fuel Cell Hybrid Electric Vehicle', Proc. IEEE Int. Conf. on Electric Machines and Drives (IEMDC), San Antonio, Texas, 15-18 May 2005, pp. 1814-1819

[15] Lee, W., Han, B.-M., and Cha, H.: 'Battery ripple current reduction in a three-phase interleaved dc-dc converter for 5kW battery charger', Proc. IEEE Energy Conversion Congress and Exposition (ECCE), San Diego, California, 17-22 Sept. 2011, pp. 3535-3540

[16] Chen, L.-R., Chu, N.-Y., Wang, C.-S., and Liang, R.-H.: 'Design of a Reflex-Based Bidirectional Converter With the Energy Recovery Function', IEEE Tr. Ind. Electr., Aug. '08, 55, (8), pp. 3022-3029

[17] Lai, J.-S., and Nelson, D. J.: 'Energy Management Power Converters in Hybrid Electric and Fuel Cell Vehicles', April 2007, Proc. of the IEEE, 95, (4), pp. 766-777

[18] Zhang, J., Lai, J.-S., Kim, R.-Y., and Yu, W.: 'High-Power Density Design of a Soft-Switching HighPower Bidirectional dc-dc Converter', IEEE Trans. Power Electronics, July 2007, 22, (4), pp. 1145-1153

[19] Staunton, R. H., Ayers, C. W., Marlino, L. D., Chiasson, J. N., and Burress, T. A.: 'Evaluation of 2004 Toyota Prius Hybrid Electric Drive System', Tech. Rep. ORNL/TM-2006/423, May 2006, Oak Ridge National Laboratory, Oak Ridge, TN, USA

[20] Tant, P., et al., 'Case-study of an Educational Engineering Project: a Series Hybrid Electric Kart', Journal sur l'enseignement des sciences et technologies de l'information et des systèmes, 2009, 8

[21] De Breucker, S., Engelen, K., Tant, P., and Driesen, J.: 'Adaptive control scheme for a practical bidirectional DC-DC converter with a $80 \mathrm{kHz}$ switching and a $10 \mathrm{kHz}$ sampling frequency', Proc. $5^{\text {th }}$ IET Int. Conf. on Power Electr., Mach. \& Drives (PEMD 2010), 19-21 April '10, Brighton, United Kingdom

[22] Mohan, N., Undeland, T.M., and Robbins, W.P.: Power Electronics: Converters, Applications and Design, 3rd ed., John Wiley \& Sons, 2003 